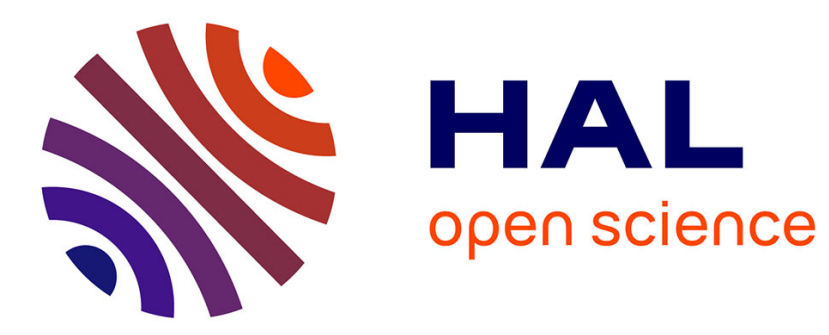

\title{
Strong nonlinear optical effects in micro-confined atmospheric air
}

Benoît Debord, Martin Maurel, Frédéric Gérôme, Luca Vincetti, Anton Husakou, Fetah Benabid

\section{- To cite this version:}

Benoît Debord, Martin Maurel, Frédéric Gérôme, Luca Vincetti, Anton Husakou, et al.. Strong nonlinear optical effects in micro-confined atmospheric air. Photonics research, 2019, 10.1364/PRJ.7.001134 . hal-02328018

\section{HAL Id: hal-02328018 https://hal.science/hal-02328018}

Submitted on 6 Jul 2020

HAL is a multi-disciplinary open access archive for the deposit and dissemination of scientific research documents, whether they are published or not. The documents may come from teaching and research institutions in France or abroad, or from public or private research centers.
L'archive ouverte pluridisciplinaire HAL, est destinée au dépôt et à la diffusion de documents scientifiques de niveau recherche, publiés ou non, émanant des établissements d'enseignement et de recherche français ou étrangers, des laboratoires publics ou privés. 


\title{
PHOTONICS Research
}

\section{Strong nonlinear optical effects in micro-confined atmospheric air}

\author{
Benoit Debord, ${ }^{1,2, *}$ Martin Maurel, ${ }^{1,2}$ Frederic Gerome, ${ }^{1,2}$ Luca Vincetti, ${ }^{3}$ Anton Husakou, ${ }^{4}$ \\ and Fetah Benabid ${ }^{1,2,5}$ \\ ${ }^{1}$ GPPMM Group, Xlim Research Institute, CNRS UMR 7252, University of Limoges, France \\ ${ }^{2}$ GLOphotonics S.A.S, 123 avenue Albert Thomas, 87060 Limoges, France \\ ${ }^{3}$ Department of Engineering "Enzo Ferrari", University of Modena and Reggio Emilia, I-41125 Modena, Italy \\ ${ }^{4}$ Max Born Institute, Max-Born-Str. 2a, D-12489 Berlin, Germany \\ ${ }^{5}$ e-mail: f.benabid@xlim.fr \\ ${ }^{*}$ Corresponding author: benoit.debord@xlim.fr
}

Received 1 April 2019; revised 18 July 2019; accepted 1 August 2019; posted 1 August 2019 (Doc. ID 362835); published 18 September 2019

\begin{abstract}
Historically, nonlinear optical phenomena such as spectral broadening by harmonic generation have been associated with crystals owing to their strong nonlinear refractive indices, which are in the range of $\sim 10^{-14} \mathrm{~cm}^{2} / \mathrm{W}$. This association was also the result of the limited optical power available from early lasers and the limited interaction length that the laser-crystal interaction architecture could offer. Consequently, these limitations disqualified a large number of materials whose nonlinear coefficient is lower than $n_{2} \sim 10^{-16} \mathrm{~cm}^{2} / \mathrm{W}$ as suitable materials for nonlinear optics applications. For example, it is a common practice in most of optical laboratories to consider ambient or atmospheric air as a "nonlinear optically" inert medium due to its very low nonlinear coefficient $\left(\sim 10.10^{-19} \mathrm{~cm}^{2} / \mathrm{W}\right)$ and low density. Today, the wide spread of high-power ultra-short pulse lasers on one hand, and low transmission loss and high-power handling of Kagome hollow-core photonic crystal fiber on the other hand, provide the necessary ingredients to excite strong nonlinear optical effects in practically any gas media, regardless of how low its optical nonlinear response is. By using a single table-top $1 \mathrm{~mJ}$ ultra-short pulse laser and an air exposed inhibited-coupling guiding hollow-core photonic crystal fiber, we observed generation of supercontinuum and third harmonic generation when the laser pulse duration was set at $600 \mathrm{fs}$ and Raman comb generation when the duration was $300 \mathrm{ps}$. The supercontinuum spectrum spans over $\sim 1000 \mathrm{THz}$ and exhibits a typical spectral-density energy of $150 \mathrm{~nJ} / \mathrm{nm}$. The dispersion profile of inhibited-coupling hollow-core fiber imprints a distinctive sequence in the supercontinuum generation, which is triggered by the generation of a cascade of four-wave mixing lines and concluded by solitonic dynamics. The Raman comb spans over $300 \mathrm{THz}$ and exhibits multiple sidebands originating from $\mathrm{N}_{2}$ vibrational and ro-vibrational Raman transitions. With the growing use of hollow-core photonic crystal fiber in different fields, the results can be applied to mitigate air nonlinear response when it is not desired or to use ambient air as a convenient nonlinear medium. () 2019 Chinese Laser Press
\end{abstract}

https://doi.org/10.1364/PRJ.7.001134

\section{INTRODUCTION}

Historically, transparent crystals and then glasses have strongly been associated with nonlinear optics [1]. Their high density and their strong laser-induced nonlinear polarization density meant a large nonlinear refractive index coefficient (typically $\left.n_{2} \sim 10^{-14}-10^{-16} \mathrm{~cm}^{2} / \mathrm{W}\right)$, enabling generation of nonlinear effects with relatively low laser power levels and short interaction length [2]. This is exemplified by the seminal demonstration of second-harmonic generation in ruby by Franken [3] and the original supercontinuum (SC) generation in borosilicate BK7 glass by Alfano and co-workers [4]. Since these early works, nonlinear optics has continuously been driven by the progress in two fields: the optical material field and the laser field. For example, research on optical materials enabled us to identify a large number of alternative nonlinear optical materials including non-solid phase materials such as liquids [5], whose nonlinear indices could be as high as those of crystals. Furthermore, the development of high-intensity and ultrashort-duration lasers enabled the demonstration of SC in high-pressure gases [6].

Another landmark in the progress of nonlinear optics is illustrated by the generation of over one-octave-wide SC in silica-core photonic crystal fibers $(\mathrm{PCFs})[2,7]$. This created a new paradigm whereby the main driving contribution to the 
enhancement of nonlinear effects is provided no longer by a new progress in lasers or materials but by the waveguide configuration of the laser-matter interaction. The extremely small effective area and long interaction length offered by this PCF configuration enabled the excitation of strong optical nonlinearities with laser peak power levels that are orders of magnitude lower than what was previously possible in the previously well-explored silica glass material [7]. This ability to generate strong optical nonlinear effects with low power levels has been extended to gases and liquids with the advent of hollow-core photonic crystal fiber $[8,9]$. Among the recent demonstrations of such strong optical nonlinearities at low light levels, we count the generation of several-octave SC [10] and Raman combs [11] in hydrogen-filled Kagome hollow-core PCF (HC-PCF). This fiber technology allows meters-long and micrometer transverse laser beam confinement with strong light/gas interaction when its core is filled with gas. The various core/cladding designs of the inhibited-coupling HC-PCF family offer a large tunability in terms of core size, dispersion value, and profile.

Following up on our previous work [12], we show in this paper that very weakly nonlinear optical materials can also be used within a simple and table-top experimental setup as a platform for the generation of strong and multifarious nonlinear optical effects. We choose atmospheric air as a material for this purpose. The choice is motivated by the fact that air is ambient and requires no special exploitation engineering, as well as by the fact that air, except for some applications in high optical field, has until now been mostly considered as a nonlinear optically inert medium due to its very low nonlinear coefficient $\left(n_{2} \sim 10.10^{-19} \mathrm{~cm}^{2} / \mathrm{W}\right)$ and its low density. Furthermore, this work is enabled and motivated by two major advances. The first advance concerns the dramatic progress in power scaling of ultra-short-pulse (USP) lasers, which followed the invention of the chirped pulse amplification (CPA) technique $[13,14]$. Today, such lasers can offer compact solutions with a large dynamic range in repetition rate spanning from kilohertz $(\mathrm{kHz})$ to megahertz $(\mathrm{MHz})$ and intensity levels in the range of petawatt per square centimeter $\left(\mathrm{PW} / \mathrm{cm}^{2}\right)$. The second advance is associated with the development of inhibitedcoupling (IC) optical guidance, which led to the seminal introduction of hypocycloid core contour (i.e., negative curvature) in hollow-core fibers [15]. Today, IC Kagome HC-PCF with hypocycloid core contour combines low loss [16-19], large optical bandwidth, and record USP energy handling reaching the millijoule $(\mathrm{mJ})$ level [20].

Here, we report on the use of a single table-top experimental setup consisting of a USP laser and two atmospheric air-filled IC Kagome fibers to generate a $300 \mathrm{THz}$ wide Raman comb with $70 \mathrm{THz}$ spacing, a $\sim 1000 \mathrm{THz}$ broad SC with a record energy spectral density of $150 \mathrm{~nJ} / \mathrm{nm}$, and third-harmonic generation. The dynamics of the SC generation are described, resulting from varied phenomena such as four-wave mixing (FWM), third-harmonic generation (THG), and soliton formation.

\section{EXPERIMENTS}

\section{A. Experimental Setup}

The experimental setup used for the different experiments reported in this paper is shown in Fig. 1. A single, table-top Yb-based CPA laser, operating at $1030 \mathrm{~nm}$, emits up to $1.36 \mathrm{~mJ}$ energy pulses at $1 \mathrm{kHz}$ repetition rate. The laser can emit either 300 ps duration pulses through a first channel labelled "output 1" in Fig. 1 or 600 fs pulses through another channel labelled "output 2." The laser beam from each channel is coupled into an IC Kagome HC-PCF.

The IC HC-PCFs were simply exposed to the ambient air. This air-filling configuration drastically simplifies the laser beam injection module that is composed just of a lens and a translation stage. The launching power is controlled by the use of two half-wave plates and a polarizing beam splitter. The air is gas mixture having a pressure of $1 \mathrm{~atm}$, with the main constituents being nitrogen molecules $\mathrm{N}_{2}$ (78\% in volume) and oxygen molecules $\mathrm{O}_{2}$ (21\% in volume) and to a lesser extent argon (1\% in volume).

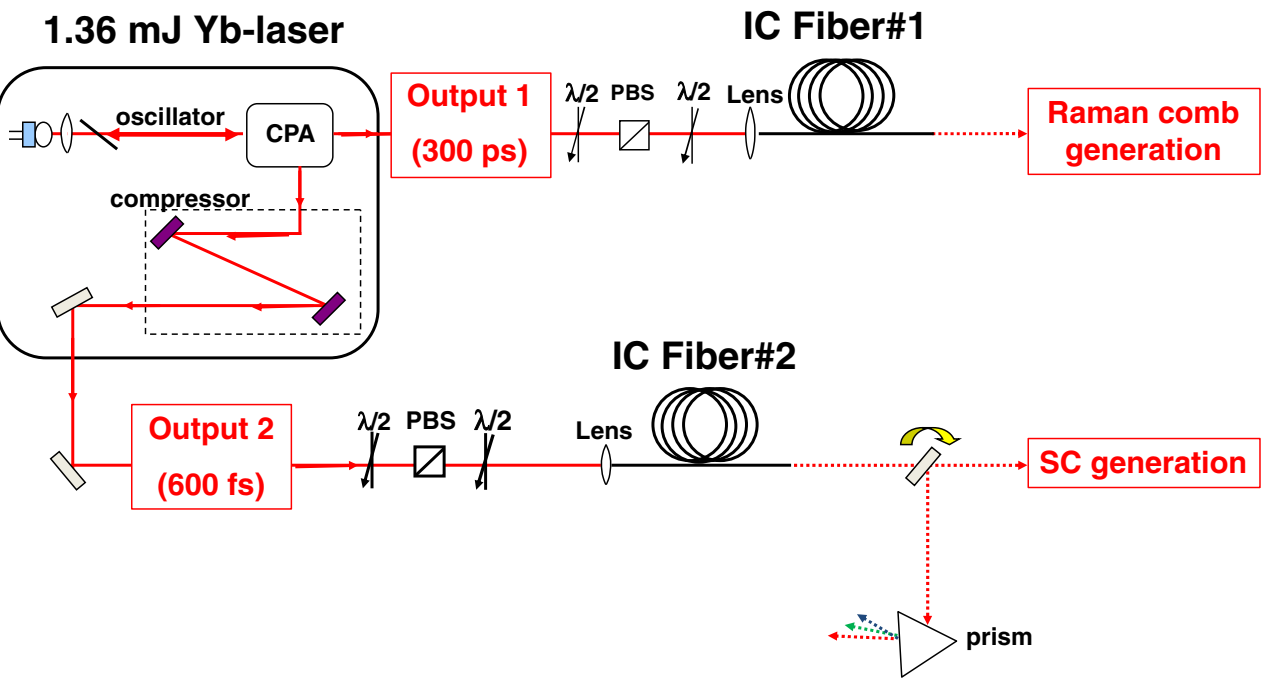

Fig. 1. Experimental setup. Table-top $\mathrm{Yb}$ laser $(1.36 \mathrm{~mJ}, 1 \mathrm{kHz}$ repetition rate) composed of two output channels ("output 1" corresponding to 300 ps pulses, and "output 2" corresponding to 600 fs pulses) coupled into two different IC Kagome HC-PCFs. 
Considering only the nitrogen and oxygen molecules, the nonlinear polarization in both molecules is based on third-order susceptibility, and it can be induced either by the electronic Kerr effect or stimulated Raman scattering (SRS). The Kerr effect leads to the index-change-induced field phase variation and manifests itself in self-phase modulation (SPM) spectral broadening, FWM, and THG. SRS process results from a resonant response of the third-order susceptibility and manifests itself via conversion of the pump frequency $v_{p}$ to discrete spectral lines with frequencies $\nu_{ \pm m}=\nu_{P} \pm m \nu_{R}$ down-shifted (Stokes lines) or up-shifted (anti-Stokes lines) by the Raman transition frequency $\nu_{R}$. Here, $m$ is a positive integer corresponding to the Raman order of the generated Stokes and anti-Stokes lines.

Table 1 summarizes the main ambient air material properties that are relevant in the laser pulse propagation nonlinear optical dynamics. The table shows that atmospheric air has a nonlinear refractive index of $n_{2} \sim 10.10^{-19} \mathrm{~cm}^{2} / \mathrm{W}[21,22]$. Furthermore, the table shows the steady-state Raman gain coefficient $g_{s s}$ for both $\mathrm{N}_{2}$ and $\mathrm{O}_{2}$. Here, $g_{s s}$ is defined from the exponential expression of the first-order Stokes intensity under steady state $I_{s}(l)=I_{0} e^{g_{s s} I_{p} l}$, where $I_{p}$ is the pump laser intensity and $l$ is the fiber length. Both molecules exhibit a vibrational $\mathrm{Q}$ (1) Raman transition with a respective resonance frequency of $69.9 \mathrm{THz}$ and $46.6 \mathrm{THz}$. These resonances have a gain coefficient $g_{s s}$ of $35 \mathrm{~cm} / \mathrm{TW}$ and $22 \mathrm{~cm} / \mathrm{TW}$, respectively, and a dephasing time $T_{2}$ of 7.1 ps for $\mathrm{N}_{2}$ and 5.9 ps for $\mathrm{O}_{2}$ [23]. Thus, one would expect the SRS process to be mainly governed by $\mathrm{N}_{2}$ molecules. The table also shows that atmospheric air nitrogen exhibits several rotational Raman lines $S(J)$ (corresponding to a transition from the rotational quantum number $J$ to $J+2$ ) with the comparable Raman resonance frequencies and gain coefficients [24]. For these transitions, the dephasing times $T_{2}$ are deduced from Ref. [25]. Similarly, $\mathrm{O}_{2}$ also has rotational Raman transitions; however, we deliberately ignore them because of the relatively low density of $\mathrm{O}_{2}$.

It is noteworthy to recall that the maximum $g_{s s}$ found in air nitrogen or oxygen is several hundred times lower than that found in hydrogen molecular gas.

Due to the broadband operation and Raman-active filling gas, the considered experimental setup supports manifestation of a number of nonlinear effects: Raman gain, self-phase modulation, FWM, and THG. The relative impact of these effects is determined by their characteristic lengths: $L_{R}, L_{\mathrm{SPM}}, L_{\mathrm{FWM}}$, and $L_{\mathrm{THG}}$, correspondingly. The shorter the characteristic length, the earlier the associated nonlinear process manifests

Table 1. Kerr and SRS Coefficients of Main Atmospheric Air Gases

\begin{tabular}{|c|c|c|c|c|c|}
\hline Molecules & $\begin{array}{c}\text { Raman } \\
\text { Frequency } \\
\text { Shift (THz) }\end{array}$ & $\left(10^{-3}\right.$ & $\begin{array}{l}g_{s s} \\
\mathrm{~cm} / \mathrm{GW})\end{array}$ & $\begin{array}{l}T_{2} \\
\text { (ps) }\end{array}$ & $\begin{array}{c}n_{2} \\
\left(10^{-19} \mathrm{~cm}^{2} / \mathrm{W}\right. \\
\text { at } 1 \mathrm{bar})\end{array}$ \\
\hline Air & & & & & 10 \\
\hline $\mathrm{N}_{2} \quad \mathrm{Q}(1)$ & 69.9 & & 35 & 7.1 & 8 \\
\hline$S(6)$ & 1.8 & & 6.3 & $\sim 200$ & \\
\hline$S(8)$ & 2.3 & & 7.3 & & \\
\hline$S(10)$ & 2.8 & & 7.2 & & \\
\hline$S(12)$ & 3.2 & & 6.1 & & \\
\hline $\mathrm{O}_{2}$ & 46.6 & & 22 & 5.9 & 30 \\
\hline
\end{tabular}

itself. In addition to the above intrinsic properties of the molecules, the competition between these four effects in air strongly depends on the pump pulse duration $\tau$ and is also influenced by the fiber dispersion as detailed below. We note parenthetically that for a fixed pump power the intensity is higher for a smaller fiber cross section, and all the characteristic lengths are correspondingly shorter; this dependence is exactly identical for all four effects.

The characteristic length for the Raman effect is given by [26-28]

$$
L_{R} \approx \frac{25}{g_{s s} R(\tau) I_{p}} .
$$

Here, the factor 25 corresponds to the necessary Raman net gain to reach the stimulated scattering. The dependence of the Raman gain correction factor $R$ on pulse duration $\tau$, the molecular dephasing rate $\Gamma$, the fiber length $L$, and the effective area $A_{\text {eff }}$ is given by

$$
R(\tau) \approx \sqrt{(\Gamma \tau) / g_{s s} L I_{p}},
$$

where $I_{p}$ is the pump intensity. One can see that Raman effect becomes slightly stronger for longer pulses and manifests itself earlier in propagation. In addition, the dynamics of the Raman effect are influenced by the dispersion due to the role of walk-off.

In order to define the characteristic length for the SPM $L_{\text {SPM }}$, we need to choose the spectral width to be reached at $L_{\mathrm{SPM}}$. For a dominating Raman effect, the Raman gain will result in generation of a clear comb with period of $\Delta \omega_{R}$ offset from the pump frequency. On the other hand, if SPM contribution is dominating over the Raman effect, the spectral broadening will extend the spectrum to $\Delta \omega_{R}$ before sidebands develop. Therefore, for our aims we define $L_{\mathrm{SPM}}$ as the length at which the spectral width reaches $2 \Delta \omega_{R}$ :

$$
L_{\mathrm{SPM}}=\frac{\lambda_{p} \tau \Delta \omega_{R}}{n_{2} I_{p}} .
$$

It is clear that the relative role of the SPM increases with decreasing duration, while the dependence of $L_{R}$ on $\tau$ is weaker. From comparison of $L_{R}$ and $L_{\mathrm{SPM}}$, we expect that SPM will be the dominating mechanism for short pulses, while for longer pulses Raman effect will take the leading role. The group velocity dispersion can significantly influence the SPM process, resulting in pulse elongation and saturation of spectral broadening for normal group velocity dispersion, or resulting in soliton dynamics and SC generation for anomalous dispersion (AD).

For FWM, the characteristic length is given by [29]

$$
L_{\mathrm{FWM}}=\frac{\lambda_{p}}{2 \pi n_{2} I_{p}} .
$$

In addition, efficient FWM is only possible if the phasematching condition $2 \beta\left(\nu_{p}\right)=\beta\left(\nu_{p}+\Delta\right)+\beta\left(\nu_{p}-\Delta\right)$ is satisfied, where $\nu_{p}+\Delta$ and $\nu_{p}-\Delta$ are the signal and idler frequencies, correspondingly.

Finally, the characteristic length for the THG $\left(3 v_{p}\right)$ is

$$
L_{\mathrm{THG}}=\frac{25 \lambda_{p}}{2 \pi n_{2} I_{p}} .
$$


For our conditions, both FWM and THG will manifest at propagation lengths longer than $L_{\mathrm{SPM}}$. However, due to the significant spectral offset from the pump frequency $\nu_{p}$, which is given by $\Delta$ for FWM and even larger value of $2 \nu_{p}$ for THG, the corresponding spectral peaks will still be discernible in the spectrum.

Table 2 provides values of characteristic lengths of the different above-mentioned nonlinear effects (Raman gain, SPM, FWM, and THG) for a representative input pulse intensity of $96 \mathrm{TW} / \mathrm{cm}^{2}$, which corresponds to an energy of $800 \mu \mathrm{J}$ at $600 \mathrm{fs}$. One can clearly see that for the shortest pulse duration (600 fs) the dominant effect is the SPM, whereas for longer pulses (300 ps) Raman effect dominates with a characteristic

Table 2. Characteristic Lengths of Nonlinear Effects: Raman Gain, Self-Phase Modulation, Four-Wave Mixing, and Third Harmonic Generation, for $96 \mathrm{TW} / \mathrm{cm}^{2}$ Input Intensity

\begin{tabular}{lcccc}
\hline Pulse Width & $\boldsymbol{L}_{\boldsymbol{R}}(\mathbf{c m})$ & $\boldsymbol{L}_{\mathrm{SPM}}(\mathbf{c m})$ & $\boldsymbol{L}_{\mathrm{FWM}}(\mathbf{c m})$ & $\boldsymbol{L}_{\mathrm{THG}}(\mathbf{c m})$ \\
\hline $600 \mathrm{fs}$ & 15 & 1.3 & $\sim 0.6$ & 15 \\
$300 \mathrm{ps}$ & 0.65 & 600 & $\sim 0.6$ & 15 \\
\hline
\end{tabular}

length of $6.5 \mathrm{~mm}$. The two other effects are dependent on phase-matching conditions and lead to an FWM length of $0.6 \mathrm{~cm}$ and a THG length of $15 \mathrm{~cm}$ for perfect phase-matching.

\section{B. HC-PCF Properties}

Both fibers used are IC guiding Kagome-lattice HC-PCFs with hypocycloid core. Figure 2(a) shows the scanning electron microscope (SEM) images of the two fibers. They are based on a sevencell core defect design with a negative curvature parameter $b$ equal to 1 (see Ref. [16] for definition of $b$ ). Their inner core radius $R_{\text {in }}$ is equal to $30 \mu \mathrm{m}$, corresponding to a mode field diameter (MFD) of $42 \mu \mathrm{m}$. However, the fibers have different silica strut thicknesses so that they provide specific transmission and dispersion spectra. This is illustrated in Figs. 2(b) and 2(c), showing the two fibers' spectra of transmission over a $4 \mathrm{~m}$ long fiber section (shaded grey curves), the transmission loss (blue curves), and the effective index of the fiber-core fundamental mode (black curves).

The fiber transmission and loss spectra exhibit a series of low-loss transmission bands separated with non-guiding bands centered at wavelengths related to the strut thickness by $\lambda_{l}=(2 t / l) \sqrt{n_{g}^{2}-1}$. Here, $t$ and $n_{g}$ are the silica-strut (a)

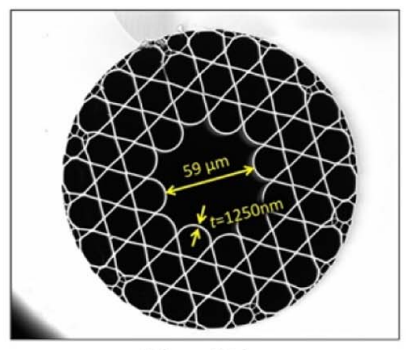

Fiber (A) (b)
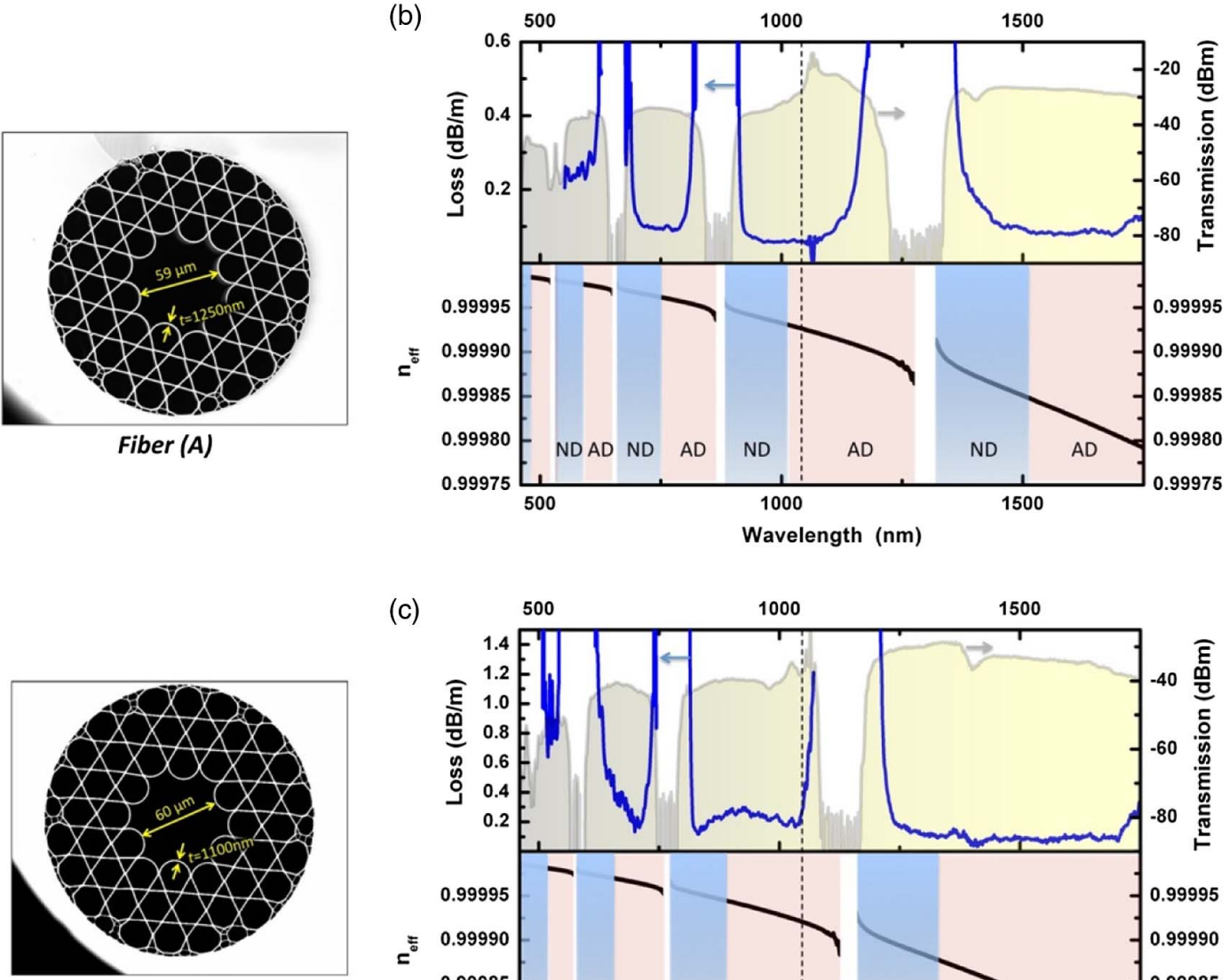

Fiber (B) (c)

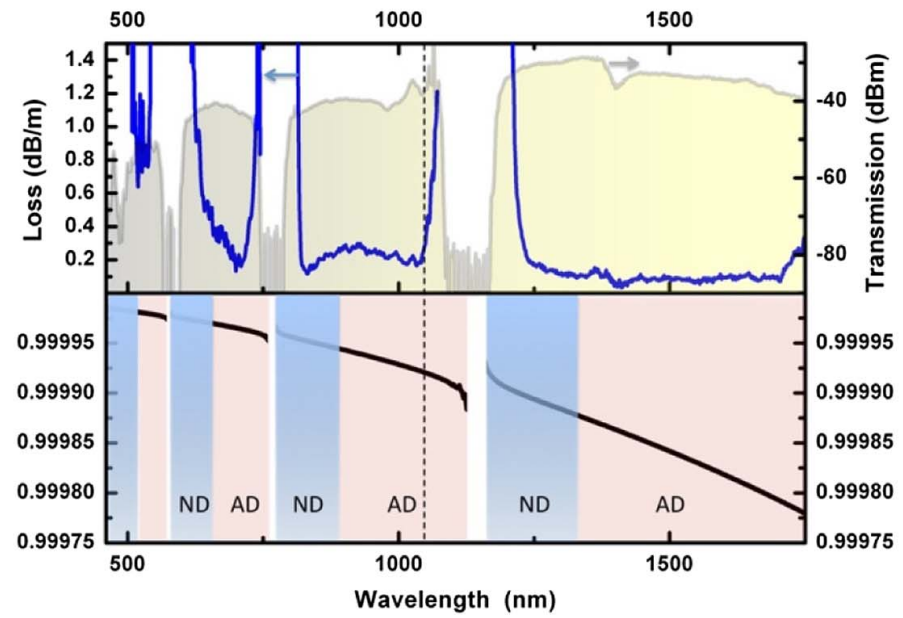

Fig. 2. (a) SEM images of the cross section of the two IC fibers used. Transmission (shaded grey curves), loss (blue curves), and effective index of the core fundamental mode (black curve) spectra for (b) Fiber \#A and (c) Fiber \#B. Normal and anomalous dispersion regimes in different transmission bands identified by ND and AD, respectively. 
thickness and index, respectively, and $l$ is a positive integer. The non-guiding bands correspond to a strong transverse-matchinginduced coupling between the core mode and the silica cladding modes [30]. Over our measurable spectral range of 400-1750 nm, Fiber \#A exhibits 5 transmission windows, its second-order transmission band exhibiting an edge centered at $1250 \mathrm{~nm}$. This is consistent with the measured strut thickness of $1250 \mathrm{~nm}$. This thickness has been chosen to optimize the number of generated Stokes lines. At the operating $1030 \mathrm{~nm}$ laser wavelength, the fiber has transmission loss of $60 \mathrm{~dB} / \mathrm{km}$. Fiber \#B has a silica-strut thickness of $1100 \mathrm{~nm}$, and accordingly its transmission spectrum exhibits transmission bands that are blue-shifted by $10 \%$ relative to those of Fiber \#A. Fiber \#B has a loss value of $200 \mathrm{~dB} / \mathrm{km}$ at $1030 \mathrm{~nm}$.

The black curves in Figs. 2(b) and 2(c) show the numerically calculated effective index of the fiber-core fundamental mode for Fiber \#A and Fiber \#B, respectively. Similar to the transmission and loss spectra, because of the coupling between the core mode with the cladding modes near the wavelengths $\lambda_{l}$, the core fundamental-mode chromatic dispersion exhibits discontinuities in the non-guiding regions with an S-like profile at each transmission band $[16,30]$. As a result, each transmission window is segmented into a normal dispersion (ND) region and $\mathrm{AD}$ region [see the shaded regions in Figs. 2(b) and 2(c)].

For the experiments developed below, a $3 \mathrm{~m}$ long piece of Fiber \#A is excited by 300 ps pulses. Here the laser wavelength (represented by dashed line in Fig. 2) is located close to the zero-group velocity dispersion (ZGVD) of the second higherorder transmission band. Furthermore, a $3.8 \mathrm{~m}$ long piece of Fiber \#B is excited by the $600 \mathrm{fs}$ pulses, and the laser wavelength is located in the $\mathrm{AD}$ region of the second higher-order transmission band.

\section{RESULTS}

\section{A. Raman Comb Generation}

Figure 3 shows the evolution of the output spectrum with the input energy in a $3 \mathrm{~m}$ long piece of Fiber \#A. The map shows the development of distinct spectral lines with a Raman comb that spans over a wide spectrum range, up to $\sim 300 \mathrm{THz}$ (from 600 to $1375 \mathrm{~nm}$ ), with a comb spacing of $70 \mathrm{THz}$ and evolving as follows.

The first two lines appear at an energy of $650 \mu \mathrm{J}$ (corresponding to a transmitted energy of $530 \mu \mathrm{J}$ ) that correspond to the first Stokes line (noted $S_{1}$ ) and first anti-Stokes line of $\mathrm{N}_{2}$ (noted $A S_{1}$ ) vibrational Raman resonance $\mathrm{Q}(1)$. The second anti-Stokes line appears for an input energy of $750 \mu \mathrm{J}$, and then another one for $1200 \mu \mathrm{J}$. The energy is mainly distributed between the pump, $S_{1}$, and $\mathrm{AS}_{1}$. We can see that each line presents a spectral feature characterized by spectral broadening increasing with the input energy, attributed in this case to the rotational response of the nitrogen (in the present graph, the S6 system). We also observe the $S 8$ lines, though not as clearly as the $S 6$ lines. This fact is due to the combination of the smaller strength of the S8 Raman transition and the resolution of the optical spectrum analyzer (OSA).

Figure 3(b) shows a zoom of the $\mathrm{AS}_{2}$ line at the maximum output energy where the different rotational lines of the second vibrational line are identified.

The graph at the top of Fig. 3 represents the generated spectrum at the maximum $1.36 \mathrm{~mJ}$ launched energy. For this input energy laser beam, an output energy of $1.03 \mathrm{~mJ}$ is measured, corresponding to an impressive $75 \%$ transmission. The evolution of the transmission coefficient with the input energy is plotted on the right side of the map. One can see that for input energies up to $700 \mathrm{~mJ}$, the transmission is equal to $83 \%$ and drops gently after the comb onset, reaching $75 \%$ for the maximum energy. This reveals that the Raman comb dynamics do not imply significant loss. Finally, the comb structure of the spectrum shows an insignificant to zero effect from Kerr nonlinearity, even at energy value as high as $1.03 \mathrm{~mJ}$, which contrasts with those obtained with 6 ps [31].

\section{B. Supercontinuum Generation}

The excitation of the $3.8 \mathrm{~m}$ long piece of Fiber \#B by $600 \mathrm{fs}$ pulse duration presents a completely different spectral dynamic.

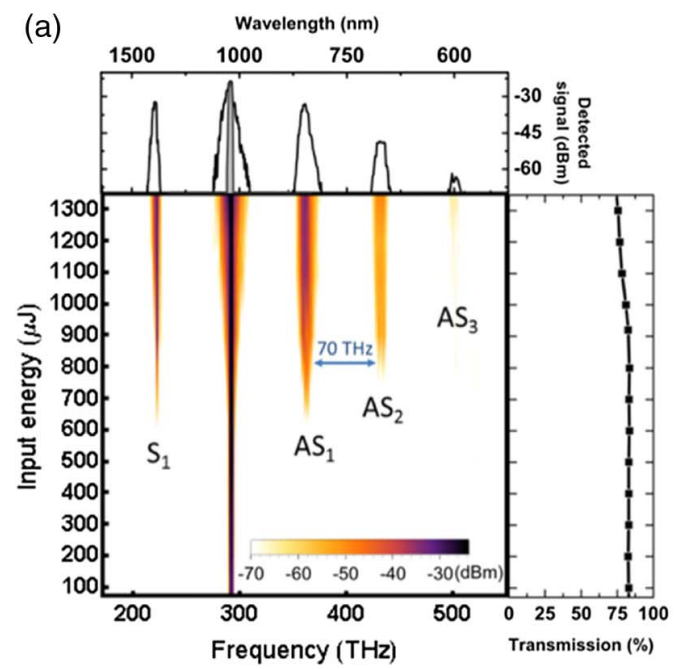

(b)

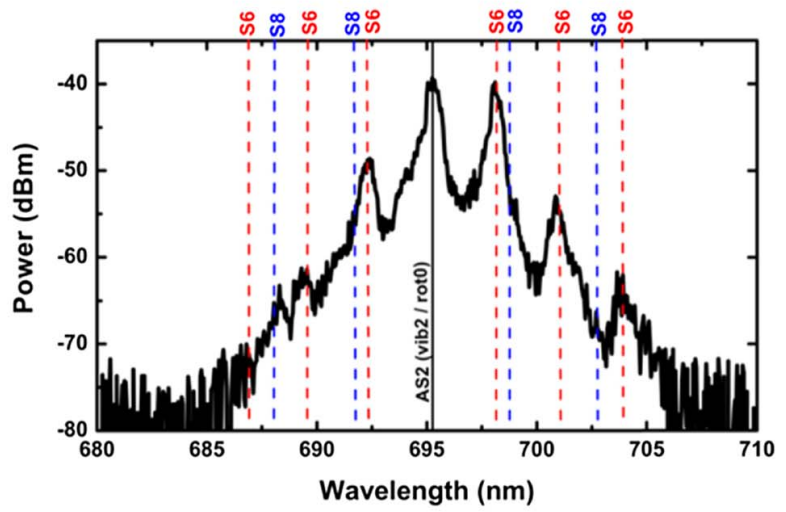

Fig. 3. (a) Raman comb generated at the output of IC Fiber \#A for a 300 ps laser pulse duration. Evolution of the $3 \mathrm{~m}$ long air-filled IC Fiber \#A output spectrum. (Top) Output spectrum for input energy of $1.36 \mathrm{~mJ}$. The input pulse is shown for comparison. (Right) Transmission coefficient versus input energy. (b) Rotational response of the $\mathrm{AS}_{2}$ line. 
(a)

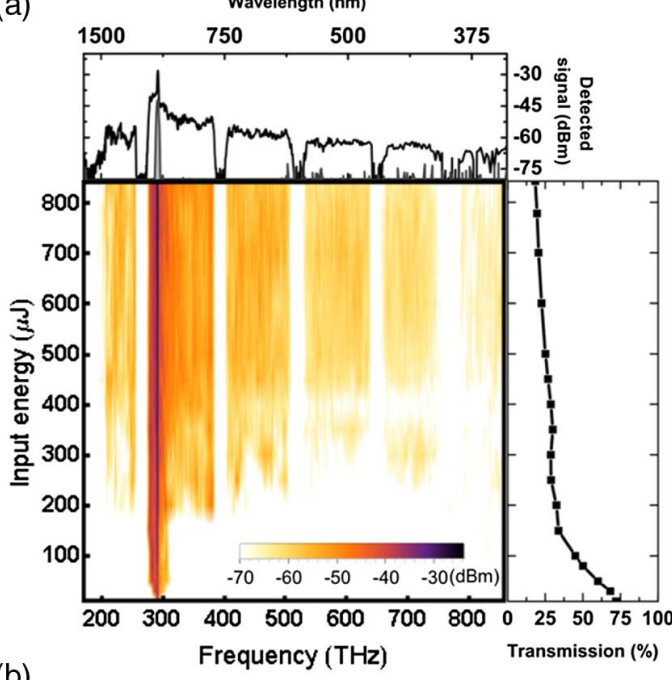

(b)

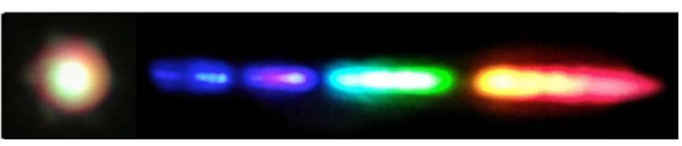

Fig. 4. (a) SC generated at the output of IC Fiber \#B for a $600 \mathrm{fs}$ laser pulse duration. Experimental evolution of the $3.8 \mathrm{~m}$ long airfilled IC Fiber \#B output spectrum. (Top) Output spectrum for input energy of $840 \mu \mathrm{J}$. The input pulse is shown for comparison. (Right) Transmission coefficient versus input energy. (b) Dispersed beam of the SC generated at the output of the IC Fiber \#B.
The peak power corresponding to this excitation is 3 orders of magnitude higher than the previously studied one, with a maximum value reaching $1.8 \mathrm{GW}$. Here, the pulse propagation dynamics are dominated by SPM and solitonic dynamics leading to a petahertz $(\sim \mathrm{PHz})$-wide SC confined within the transmission bands of the HC-PCF. The right-hand side of the map in Fig. 4 shows the transmission coefficient after the $3.8 \mathrm{~m}$-long piece. At the $\mathrm{SC}$ onset, the transmission efficiency drops from $70 \%$ to $30 \%$.

At input energy of $840 \mu \mathrm{J}$, the output energy of the SC was measured to be $155 \mu \mathrm{J}$. The corresponding energy spectral density reached a record value of $150 \mathrm{~nJ} / \mathrm{nm}$, which is 2 orders of magnitude higher than that of a SC based on fiber laser sources [32].

The dynamics of the SC generation have been studied theoretically by carrying out numerical simulations using the generalized nonlinear Schrödinger equation, augmented by the equations describing the dynamics of the Raman polarization. The group velocity dispersion to all orders, the wavelengthdependent loss, the Kerr effect, the Raman scattering, and the quantum noise were included in the simulation. Figure 5 shows the theoretical evolution with the input energy of the output spectrum for the case of a fiber length of $20 \mathrm{~cm}$. This length has been chosen to clearly see and identify the multiple processes responsible before the $\mathrm{SC}$ onset. The laser pump first induces a generation of the third harmonic at $343 \mathrm{~nm}$ under nonphase-matched conditions. However, this THG has not been (a)

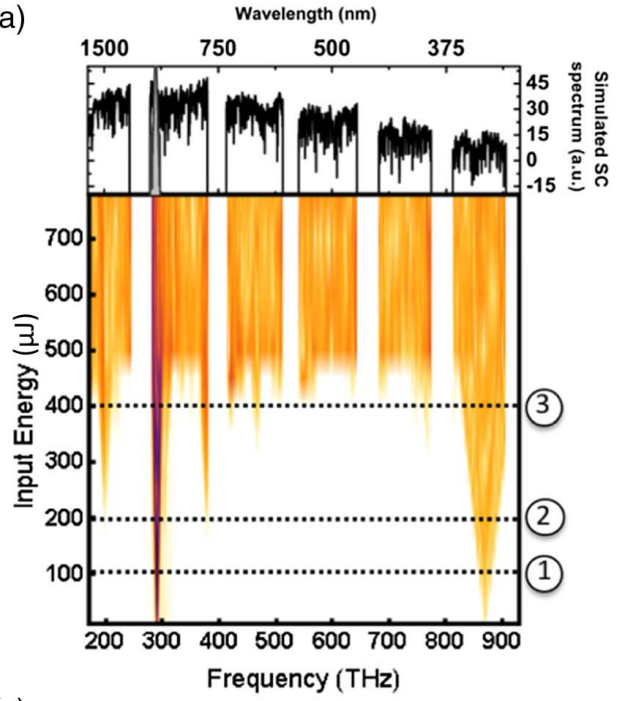

(c)

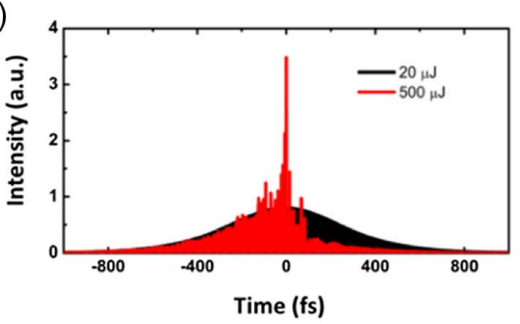

(b)

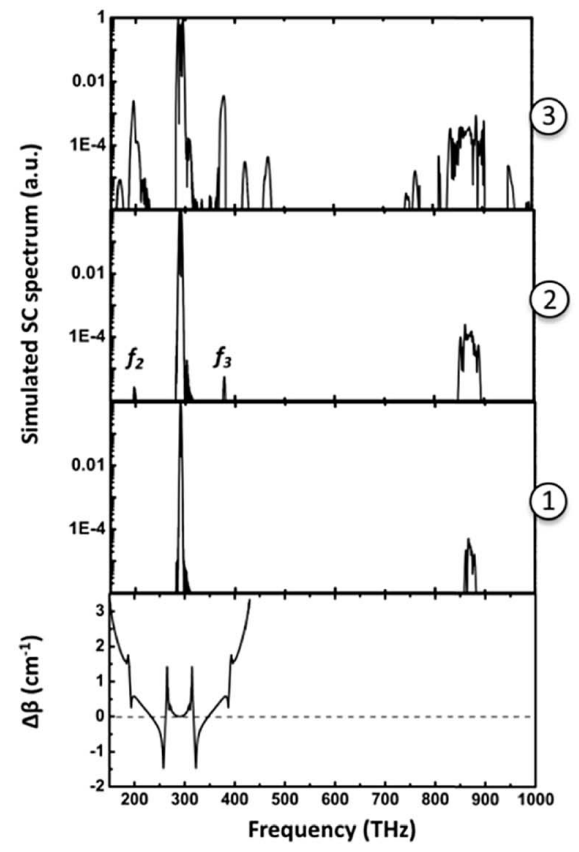

Fig. 5. (a) Theoretical spectral evolution of the $20 \mathrm{~cm}$ long air-filled IC Fiber \#B output spectrum. (Top) Output spectrum for input energy of $840 \mu \mathrm{J}$. The input pulse is shown for comparison. (b) Output fiber theoretical spectral evolution with the input energy $(100,200$, and $400 \mu \mathrm{J})$ for a fiber length of $20 \mathrm{~cm}$. (Bottom) Phase mismatch curve $\Delta \beta$ as a function of the idler/signal wavelengths $\left(f_{2}\right.$ and $\left.f_{3}\right)$. (c) Theoretical temporal pulse profiles for two input energies: $20 \mu \mathrm{J}$ and $500 \mu \mathrm{J}$. 
observed experimentally on the fundamental mode but on a higher-order one (see the next section). Then (at $200 \mu \mathrm{J}$ ) two new frequencies (denoted by $f_{2}$ and $f_{3}$ ) appear through the FWM process. These new frequencies act as a seed to create a cascade of FWM combination, allowing access to all of the transmission bands of the fiber due to the specific evolution of the effective index of the core mode [graph at the bottom of Fig. 5(b)].

After the development of the FWM sidebands, at higher energy than $400 \mu \mathrm{J}$, the temporal profile becomes highly modulated as shown in Fig. 5(c). It features many isolated spikes in the temporal profile, which remain roughly unchanged with further propagation. We have evaluated the spectral contents of the strongest of these spikes and found that its central frequency lies around the pump frequency in the $\mathrm{AD}$ region. Therefore, we conclude that the spikes have a solitonic nature, and the late stage of the SC generation is governed by soliton dynamics and possibly by the associated generation of the non-solitonic radiation.

The phase mismatch will additionally strongly influence the efficiency of both the FWM and the THG. In Fig. 5(b), we present the phase mismatch $\Delta \beta$ as a function of the idler/signal wavelength considering the fundamental core mode as

$$
\Delta \beta=2 \beta_{\mathrm{FM}}\left(f_{p}\right)-\left[\beta_{\mathrm{FM}}\left(f_{2}\right)+\beta_{\mathrm{FM}}\left(f_{3}\right)\right],
$$

where $\beta_{\mathrm{FM}}=n_{\text {eff }} k$, with $k$ the wavenumber and $n_{\text {eff given }}$ by Ref. [33].

One can see that the position of the phase-matched wavelengths is mainly defined by the transmission band edges. In addition, the contribution of the nonlinear term in the phase mismatch is significant, changing the number of the phasematching wavelength and shifting it.

\section{High Harmonic Generation}

The last phenomenon that has been explored is the generation of the third harmonic. First, the conditions of phase matching between the fundamental core mode and high-order modes have been studied theoretically [see Fig. 6(a)]. Since the pump beam is characterized by azimuthal symmetry, the third-harmonic polarization induced by pump light is also azimuthally symmetric; therefore, only phase matching between the fundamental $\mathrm{HE}_{11}$ mode and azimuthally symmetric $\mathrm{HE}_{1 n}$ modes was considered. To evaluate the effective refractive index $n_{\text {eff }}\left(3 f_{p}\right)$ of IC Kagome fiber at the third-harmonic frequency, we consider for this section the dispersion curves of $\mathrm{HE}_{11}, \mathrm{HE}_{12}, \mathrm{HE}_{13}$, and $\mathrm{HE}_{14}$ of Fiber $\# \mathrm{~B}$ using analytical formula for capillaries by Zeisberger et al. [33].

The phase mismatch $\Delta \beta_{1 n}$ between the fundamental mode and $\mathrm{HE}_{1 n}$ for THG can be written as

$$
\Delta \beta_{1 n}=\beta_{1 n}\left(3 f_{p}\right)-3 \beta_{\mathrm{FM}}\left(f_{p}\right)+3 \gamma \kappa_{1 n} P_{\text {peak }},
$$

where $\beta_{1 n}$ are propagation constants of a high-order mode, $\gamma$ is the nonlinear coefficient, $\kappa_{1 n}$ are the cross-phase-modulation overlap coefficients, and $P_{\text {peak }}$ is the peak power.

Figure 6(a) shows dependence of the phase mismatch $\Delta \beta_{1 n}$ on mode number $n$. Note that in our case this mismatch is weakly dependent on the peak power. One can see that the smallest mismatch is predicted for the $\mathrm{HE}_{12}$ and $\mathrm{HE}_{13}$ modes. The radial distribution of intensity for the $\mathrm{HE}_{12}$ mode is characterized by zero intensity at $0.44 R_{\text {in }}$ and at $R_{\text {in }}$, while distribution of intensity for the $\mathrm{HE}_{13}$ mode has three zeros: at $0.28 R_{\text {in }}$, at $0.64 R_{\text {in }}$, and at $R_{\text {in }}$.

The THG has been experimentally observed and studied as follows. The input pulses from the $1030 \mathrm{~nm}$ laser were coupled in a $30 \mathrm{~cm}$ long piece of Fiber $\# \mathrm{~B}$, and the polarization was controlled by a half-wave plate to maximize the coupling efficiency. The conversion of the pump has been measured for an input power from 5 to $175 \mathrm{~mW}$, and the output fiber spectral evolution around $343 \mathrm{~nm}$ is shown in Fig. 6(b). We observe the onset of THG at $P_{a}=115 \mathrm{~mW}$ and increase of the TH intensity between 155 and $175 \mathrm{~mW}$. The inset picture at the top left of Fig. 6(b) shows the observed light at a distance of $20 \mathrm{~cm}$ from the output tip of the fiber. The intensity profile of the observed mode is a mixture of the profiles of the $\mathrm{HE}_{12}$ and $\mathrm{HE}_{13}$ modes, in agreement with the theory developed above. We interpret the slight azimuthal modulation of the intensity (a)

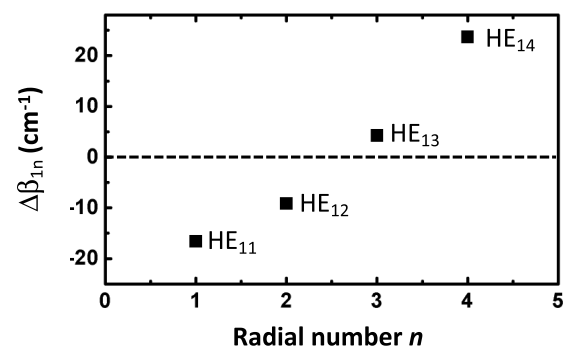

(b)

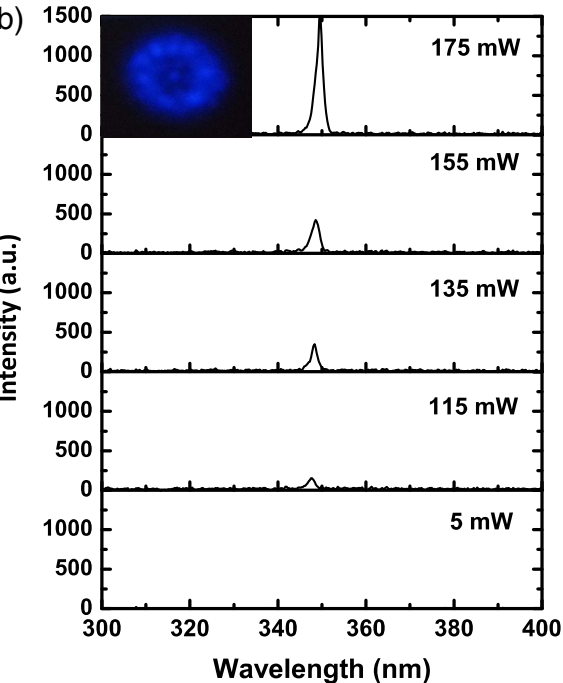

Fig. 6. (a) Phase mismatch $\Delta \beta_{1 n}$ versus the mode number $n$. (b) Experimental spectral evolution with input power, up to $175 \mathrm{~mW}$, of the THG at the output of a $30 \mathrm{~cm}$ long air-filled IC Fiber \#B. 
profile as a result of competing cascaded processes related to FWM.

\section{CONCLUSIONS}

We have experimentally demonstrated the generation of several nonlinear processes in air-filled IC HC-PCF. By using the pump pulse durations of $600 \mathrm{fs}$ and $300 \mathrm{ps}$, we have observed an intense and broad SC and a Raman comb, respectively. Finally, THG at $343 \mathrm{~nm}$ has been measured for $600 \mathrm{fs}$ input pulses. These experimental results have been supported and corroborated by theoretical results.

Funding. BPI via PIA-4F project; Agence Nationale de la Recherche (ANR) (PhotoSynth, Labex SigmaLim, UVfactor); Région Nouvelle Aquitaine, Air Force Office of Scientific Research (AFOSR) (FA9+550-14-1-0024); National Science Formation (NSF) (PHY-1068865).

Acknowledgment. The authors thank the PLATINOM (Xlim) platform for their help in the fiber fabrication.

\section{REFERENCES}

1. R. W. Boyd, Nonlinear Optics (Academic Press, 2008).

2. J. K. Ranka, R. S. Windeler, and A. J. Stentz, "Visible continuum generation in air-silica microstructure optical fibers with anomalous dispersion at $800 \mathrm{~nm}$," Opt. Lett. 25, 25-27 (2000).

3. P. A. Franken, A. E. Hill, C. W. Peters, and G. Weinreich, "Generation of optical harmonics," Phys. Rev. Lett. 7, 118-119 (1961).

4. R. R. Alfano and S. L. Shapiro, "Emission in the region 4000 to $7000 \AA$ via four-photon coupling in glass," Phys. Rev. Lett. 24, 584-587 (1970).

5. W. Werncke, A. Lau, M. Pfeiffer, K. Lenz, H.-J. Weigmann, and C. D. Thuy, "An anomalous frequency broadening in water," Opt. Commun. 4, 413-415 (1972).

6. P. B. Corkum, C. Rolland, and T. Srinivasan-Rao, "Supercontinuum generation in gases," Phys. Rev. Lett. 57, 2268-2271 (1986).

7. J. Dudley, G. Genty, and S. Coen, "Supercontinuum generation in photonic crystal fiber," Rev. Mod. Phys. 78, 1135-1184 (2006).

8. F. Benabid, J. C. Knight, G. Antonopoulos, and P. St.J. Russell, "Stimulated Raman scattering in hydrogen-filled hollow-core photonic crystal fiber," Science 298, 399-402 (2002).

9. F. Benabid and P. J. Roberts, "Linear and nonlinear optical properties of hollow core photonic crystal fiber," J. Mod. Opt. 58, 87-124 (2011).

10. F. Belli, A. Abdolvand, W. Chang, J. C. Travers, and P. St.J. Russell, "Vacuum-ultraviolet to infrared supercontinuum in hydrogen-filled photonic crystal fiber," Optica 2, 292-300 (2015).

11. A. Benoît, B. Beaudou, M. Alharbi, B. Debord, F. Gérôme, F. Salin, and F. Benabid, "Over-five octaves wide Raman combs in high-power picosecond-laser pumped $\mathrm{H}_{2}$-filled inhibited coupling Kagome fiber," Opt. Express 23, 14002-14009 (2015).

12. B. Debord, F. Gérôme, C. Honninger, E. Mottay, A. Husakou, F. Benabid, F. Benabid, and F. Benabid, "Milli-Joule energy-level comb and supercontinuum generation in atmospheric air-filled inhibited coupling Kagome fiber," in CLEO: 2015 Postdeadline Paper Digest (OSA, 2015), paper JTh5C.4.

13. D. Strickland and G. Mourou, "Compression of amplified chirped optical pulses," Opt. Commun. 55, 447-449 (1985).

14. M. D. Perry and G. Mourou, "Terawatt to petawatt subpicosecond lasers," Science 264, 917-924 (1994).
15. Y. Wang, F. Couny, P. J. Roberts, and F. Benabid, "Low loss broadband transmission in optimized core-shape Kagome hollow-core PCF," in Conference on Lasers and Electro-Optics (OSA, 2010), paper CPDB4.

16. B. Debord, M. Alharbi, T. Bradley, C. Fourcade-Dutin, Y. Y. Wang, L. Vincetti, F. Gérôme, and F. Benabid, "Hypocycloid-shaped hollowcore photonic crystal fiber Part I: arc curvature effect on confinement loss," Opt. Express 21, 28597-28608 (2013).

17. T. D. Bradley, Y. Wang, M. Alharbi, B. Debord, C. Fourcade-Dutin, B. Beaudou, F. Gerome, and F. Benabid, "Optical properties of low loss $(70 \mathrm{~dB} / \mathrm{km})$ hypocycloid-core Kagome hollow core photonic crystal fiber for $\mathrm{Rb}$ and Cs based optical applications," J. Lightwave Technol. 31, 2752-2755 (2013).

18. B. Debord, M. Alharbi, A. Benoît, D. Ghosh, M. Dontabactouny, L. Vincetti, J.-M. Blondy, F. Gérôme, and F. Benabid, "Ultra low-loss hypocycloid-core Kagome hollow-core photonic crystal fiber for green spectral-range applications," Opt. Lett. 39, 6245-6248 (2014).

19. B. Debord, A. Amsanpally, M. Chafer, A. Baz, M. Maurel, J. M. Blondy, E. Hugonnot, F. Scol, L. Vincetti, F. Gérôme, and F. Benabid, "Ultralow transmission loss in inhibited-coupling guiding hollow fibers," Optica 4, 209-217 (2017).

20. B. Debord, M. Alharbi, L. Vincetti, A. Husakou, C. Fourcade-Dutin, C. Hoenninger, E. Mottay, F. Gérôme, and F. Benabid, "Multi-meter fiber-delivery and pulse self-compression of milli-Joule femtosecond laser and fiber-aided laser-micromachining," Opt. Express 22, 1073510746 (2014).

21. E. T. J. Nibbering, G. Grillon, M. A. Franco, B. Prade, and A. Mysyrowicz, "Determination of the inertial contribution to the nonlinear refractive index of air, $\mathrm{N}_{2}$, and $\mathrm{O}_{2}$ by use of unfocused high-intensity femtosecond laser pulses," J. Opt. Soc. Am. B 14, 650-660 (1997).

22. V. Loriot, E. Hertz, O. Faucher, and B. Lavorel, "Measurement of high order Kerr refractive index of major air components," Opt. Express 17, 13429-13434 (2009).

23. A. Laubereau and W. Kaiser, "Vibrational dynamics of liquids and solids investigated by picosecond light pulses," Rev. Mod. Phys. 50, 607-665 (1978).

24. M. Rokni and A. Flusberg, "Stimulated rotational Raman scattering in the atmosphere," IEEE J. Quantum Electron. 22, 1102-1108 (1986).

25. D. R. Miller and R. P. Andres, "Rotational relaxation of molecular nitrogen," J. Chem. Phys. 46, 3418-3423 (1967).

26. F. Benabid, G. Antonopoulos, J. C. Knight, and P. St.J. Russell, "Stokes amplification regimes in quasi-cw pumped hydrogen-filled hollow-core photonic crystal fiber," Phys. Rev. Lett. 95, 213903 (2005).

27. R. J. Heeman and H. P. Godfried, "Gain reduction measurements in transient stimulated Raman scattering," IEEE J. Quantum Electron. 31, 358-364 (1995).

28. G. P. Agrawal, "Chapter 8-Stimulated Raman scattering," in Nonlinear Fiber Optics, 5th ed. (Elsevier, 2013), pp. 295-352.

29. G. P. Agrawal, "Chapter 10-Four-wave mixing," in Nonlinear Fiber Optics, 5th ed. (Elsevier, 2013), pp. 397-456.

30. F. Couny, F. Benabid, P. J. Roberts, P. S. Light, and M. G. Raymer, "Generation and photonic guidance of multi-octave optical-frequency combs," Science 318, 1118-1121 (2007).

31. S. A. Mousavi, H. C. H. Mulvad, N. Wheeler, P. Horak, T. D. Bradley, S. Alam, J. Hayes, S. R. Sandoghchi, D. Richardson, and F. Poletti, "Exploring nonlinear pulse propagation, Raman frequency conversion and near octave spanning supercontinuum generation in atmospheric air-filled hollow-core Kagomé fiber," Proc. SPIE 10088, 100880G (2017).

32. http://www.fianium.com.

33. M. Zeisberger and M. A. Schmidt, "Analytic model for the complex effective index of the leaky modes of tube-type anti-resonant hollow core fibers," Sci. Rep. 7, 11761 (2017). 